\title{
A mechanical model to investigate the role of the nucleus during confined cell migration
}

\author{
R. Allena ${ }^{a}$, H. Thiam ${ }^{b}$, M. Piel ${ }^{b}$ and D. Aubry \\ ${ }^{a}$ Arts et Metiers ParisTech, LBM, Paris, France; 'bnstitut Curie, Paris, France; 'Laboratoire MSSMat, Ecole Centrale Paris, Châtenay-Malabry, France
}

KEYWORDS Confined cell migration; continuum mechanics; computational mechanics; nucleus

\section{Introduction}

Cell migration in confinement plays a fundamental role in biological processes such as embryogenesis, immune response and tumorogenesis. Specifically, tumor cells continuously adapt their migratory behaviour to their environment. Therefore, it has become timely and essential for diagnostic purposes to quantitatively evaluate the cell deformability in confinement.

Here, we propose a two-dimensional mechanical model to simulate the migration of a HeLa cell through a microchannel. We will evaluate both the invasiveness of the cell and the mechanical forces exerted by the cell according to the surrounding microstructure.

\section{Methods}

HeLa cells are human cells with a rather rounded initial shape and a diameter of about $15 \mu \mathrm{m}$ (Ronot et al. 2000; Ngalim et al. 2013). For the numerical model, the geometry of the cell has been simplified by a circular domain $\Omega_{\text {cell }}$ of radius $r_{\text {cell }}$ (Figure 1(a)). Here, we consider two main components of the cell: the cytoplasm $\left(\Omega_{\text {cytoplasm }}\right)$ and the nucleus $\left(\Omega_{\text {nucleus }}\right)$ (Figure 1(a)). Additionally, the cell cyclically generates a frontal $\left(\Omega_{\mathrm{f}}\right)$ and a rear $\left(\Omega_{\mathrm{r}}\right)$ adhesion region in order to move forward (Allena \& Aubry 2012) (Figure 1(b)).

Both the nucleus and the cytoplasm are assumed to be viscoelastic materials and their behaviour is described by two standard Maxwell models. On one hand, the nucleus is composed by the nuclear lamina $\Omega_{\text {lamina }}$, which surrounds the viscoelastic nucleoplasm $\Omega_{\text {nucleoplasm }}$. On the other hand, the cytoplasm is essentially made of a solid phase represented by the in which the organelles such as the actin filaments are embedded. As in our previous works (Allena \& Aubry 2012; Allena 2013), we assume that the $\Omega_{\text {cytosol }}$ polymerization of the actin filaments inside the cytosol, which mostly occurs at the front of the cell (Schaub et al. 2007), generates the protrusive force at the leading edge and their contraction due to binding of myosin generates the contractile stress at the rear of the cell (Mogilner 2009).
Such active strains triggering the deformability of the cell are then described through a deformation tensor $\boldsymbol{F}_{\text {cytosol, a }}$ in the fluid-like branch of the symbolic standard Maxwell model of the cytoplasm.

As described in (Allena \& Aubry 2012; Allena 2013), the global equilibrium of the system is expressed as:

$$
\rho \mathbf{a}=\operatorname{Div}_{\mathrm{p}}\left(J \boldsymbol{\sigma} \boldsymbol{F}^{-T}\right)+f_{\mathrm{adh}}+f_{\text {channel }}
$$

where $\rho$ is the cell density, $a$ is the acceleration $\operatorname{Div}_{\mathrm{p}}$ is the divergence with respect to the initial position $p$, $J$ is the determinant of the deformation cell cortex $\Omega_{\text {cortex }}$ and a fluid-like phase, the cytosol gradient $F$ and $A^{-\mathrm{T}}$ denotes the inverse transpose of the matrix $A$ (Holzapfel 2000; Taber 2004).

$f_{\text {adh }}$ and $f_{\text {channel }}$ indicate respectively the viscous adhesion forces between the cell and the substrate and the viscous force exerted by the channel on the cell boundaries. The microchannel domain $\Omega_{\text {channel }}$ is represented by two pseudo-elliptical rigid walls with no top roof. We have tested several microchannel dimensions $(16,12,7$ and $4 \mu \mathrm{m})$ and geometries, some of which reproduce a specific experimental set up (Heuzé et al. 2011) (Figure 2 left).

\section{Results and discussion}

We have been able to define the necessary conditions in order for the cell to be invasive (i.e. able to enter the microchannel but stuck in the middle, Figure 2(c)) or permeative (i.e. able to reach the opposite side of the microchannel, Figure 2(a) and (b)). In the first case, two main conditions must be satisfied: (i) the cell protrusion length in the channel has to be larger than half the channel width and simultaneously (ii) the cell-substrate surface force must be higher than the cell-channel surface force so that the cell is able to pull on the substrate and enter into the channel. To give an example, for a sub-cellular microchannel the cell generates an adhesion force of 10 Pa versus a contact force with the microchannel of $3 \mathrm{~Pa}$. 


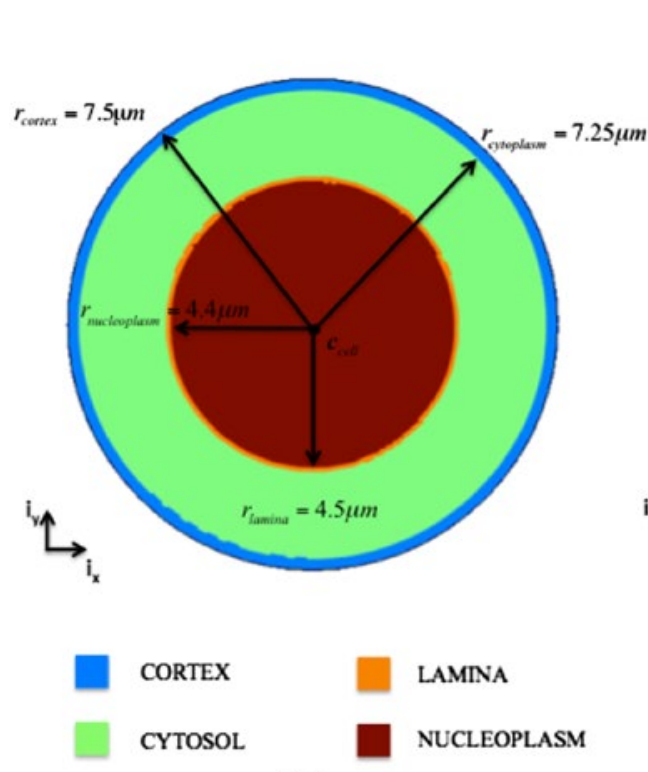

(a)

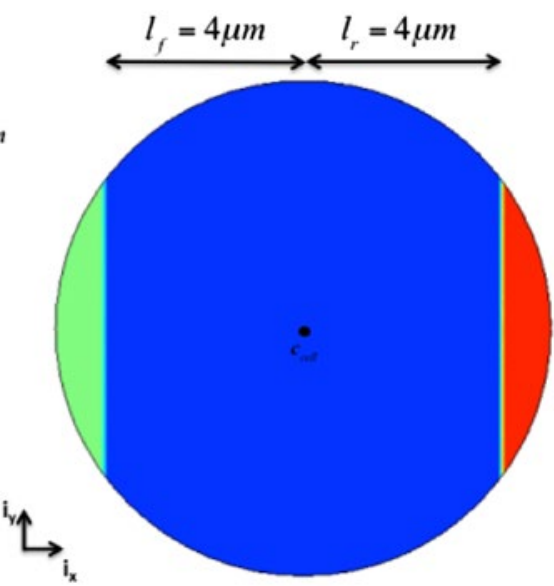

$\Omega_{r}$

$\Omega_{f}$

(b)

Figure 1. (a) Geometry of the cell and (b) frontal and rear adhesion surfaces.

(a)
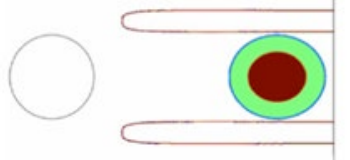

(b)

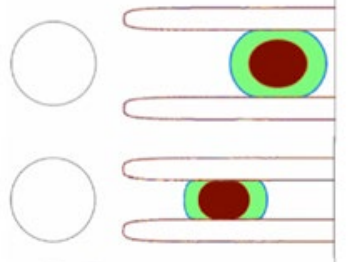

(d)

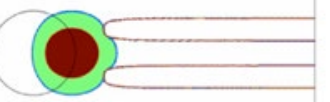

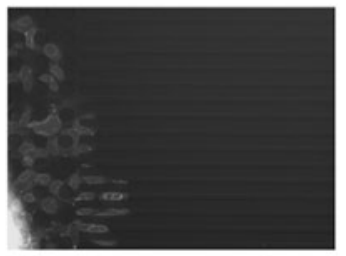

(e)

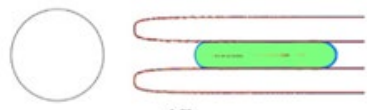

(f)

Figure 2. (a-d) Numerical simulations of confined cell migration, (e) Experimental observations for HeLa cells crossing microchannels, (f) Numerical simulation of confined migration through a subnuclear microchannel with reduced Young modulus for the nuclear lamina (Aubry et al. 2015).

For the permeative behaviour to occur, a further condition must be satisfied that is the cell-channel surface force during the whole migration has to be maximal along the upper and lower central boundaries of the cell. Those boundaries may come very close or directly in contact with the cell nucleus, which is the stiffest component of the system. Then, a larger force is required to maintain the squeezed cell shape. When the cell is stuck at the entrance of the microchannel (Figure 2(d)), it shows a penetrating behaviour. In a second series of simulations, we have shown that by reducing the modifying the mechanical properties of the nuclear lamina (i.e. reducing the Young modulus), the cell is able to achieve the opposite side of the microchannel (Figure 2(f)). The results have been compared with experimental observations on HeLa cells showing a good agreement (Figure 2(e)).

Specifically, in Figure 2 right a top view of the successive phases of the migration across a slightly subnuclear microchannel is proposed. The cell displays an invasive behaviour as it is stuck in the middle of the microchannel.

\section{References}

Allena R. 2013. Cell migration with multiple pseudopodia: temporal and spatial sensing models. Bull Math Biol. 75:288-316.

Allena R, Aubry D. 2012. 'Run-and-tumble' or 'look-and-run'? A mechanical model to explore the behavior of a migrating amoeboid cell. J Theor Biol. 306:15-31.

Aubry D, Thiam H, Piel M, Allena R. 2015. A computational mechanics approach to assess the link between cell morphology and forces during confined migration. Biomech Model Mechanobiol. 14:143-157.

Heuzé ML, Collin O, Terriac E, Lennon-Duménil A-M, Piel M. 2011. Cell migration in confinement: a microchannel-based assay. Methods Mol Biol. 769:415-434.

Holzapfel GA. 2000. Nonlinear solid mechanics: a continuum approach for engineering. 1st ed. Wiley.

Mogilner A. 2009. Mathematics of cell motility: have we got its number? J Math Biol. 58:105-134.

Ngalim SH, Magenau A, Zhu Y, Tønnesen L, Fairjones Z, Gooding JJ, Böcking T, Gaus K. 2013. Creating adhesive and soluble gradients for imaging cell migration with fluorescence microscopy. J Vis Exp: JoVE.

Ronot X, Doisy A, Tracqui P. 2000. Quantitative study of dynamic behavior of cell monolayers during in vitro wound healing by optical flow analysis. Cytometry. 41:19-30.

Schaub S, Bohnet S, Laurent VM, Meister J-J, Verkhovsky AB. 2007. Comparative maps of motion and assembly of filamentous actin and myosin II in migrating cells. Mol Biol Cell. 18:3723-3732.

Taber LA. 2004. Nonlinear theory of elasticity: applications in biomechanics. World Scientific Pub. 